\title{
The Monge-Ampère Equation for Strictly $(n-1)$-convex Functions with Neumann Condition
}

\section{Bin Deng*}

Department of Mathematics, University of Science and Technology of China, Hefei

230026, China.

Received March 10, 2019; Accepted May 14, 2019;

Published online February 28, 2020

\begin{abstract}
A $C^{2}$ function on $\mathbb{R}^{n}$ is called strictly $(n-1)$-convex if the sum of any $n-1$ eigenvalues of its Hessian is positive. In this paper, we establish a global $C^{2}$ estimates to the Monge-Ampère equation for strictly $(n-1)$-convex functions with Neumann condition. By the method of continuity, we prove an existence theorem for strictly $(n-1)$-convex solutions of the Neumann problems.
\end{abstract}

AMS subject classifications: 35J60, 35A09

Key words: Neumann problem, $(n-1)$-convex, elliptic equation.

\section{Introduction}

Let $\Omega \subset \mathbb{R}^{n}$ be a bounded convex domain and $v(x)$ be the outer unit normal at $x \in \partial \Omega$. Suppose $f \in C^{2}(\Omega)$ is positive and $\phi \in C^{3}(\bar{\Omega})$. In this paper, we mainly consider the following equations of Monge-Ampère type with Neumann condition,

$$
\begin{cases}\operatorname{det}(W)=f(x), & \text { in } \Omega, \\ \frac{\partial u}{\partial v}=-u+\phi(x), & \text { in } \quad \partial \Omega .\end{cases}
$$

where the matrix $W=\left(w_{\alpha_{1} \cdots \alpha_{m}, \beta_{1} \cdots \beta_{m}}\right)_{n \times n}$, for $m=n-1$, with the elements as follows,

$$
w_{\alpha_{1} \cdots \alpha_{m}, \beta_{1} \cdots \beta_{m}}=\sum_{i=1}^{m} \sum_{j=1}^{n} u_{\alpha_{i}} \delta_{\beta_{1} \cdots \beta_{i-1} \beta_{i} \beta_{i+1} \cdots \beta_{m}}^{\alpha_{1} \cdots \alpha_{i-1} j \alpha_{i+1} \cdots \alpha_{m}}
$$

a linear combination of $u_{i j}$, where $u_{i j}=\frac{\partial^{2} u}{\partial x_{i} \partial x_{j}}$ and $\delta_{\beta_{1} \cdots \beta_{i-1} \beta_{i} \beta_{i+1} \cdots \beta_{m}}^{\alpha_{1} \cdots \alpha_{i-1} \gamma \alpha_{i+1} \cdots \alpha_{m}}$ is the generalized Kronecker symbol. All indexes $i, j, \alpha_{i}, \beta_{i}, \cdots$ come from 1 to $n$.

${ }^{*}$ Corresponding author. Email address: bingomat@mail .ustc.edu.cn (B. Deng) 
For general $1 \leq m \leq n-1$, the matrix $W \in \mathbb{R}_{n}^{C_{n}^{m} \times C_{n}^{m}}, C_{n}^{m}=\frac{n !}{m !(n-m) !}$, comes from the following operator $U^{[m]}$ as in [2] and [10]. First, note that $\left(u_{i j}\right)_{n \times n}$ induces an operator $U$ on $\mathbb{R}^{n}$ by

$$
U\left(e_{i}\right)=\sum_{j=1}^{n} u_{i j} e_{j}, \quad \forall 1 \leq i \leq n,
$$

where $\left\{e_{1}, e_{2}, \cdots, e_{n}\right\}$ is the standard orthogonal basis of $\mathbb{R}^{n}$. We further extend $U$ to act on the real vector space $\wedge^{m} \mathbb{R}^{n}$ by

$$
U^{[m]}\left(e_{\alpha_{1}} \wedge \cdots \wedge e_{\alpha_{m}}\right)=\sum_{i=1}^{m} e_{\alpha_{1}} \wedge \cdots \wedge U\left(e_{\alpha_{i}}\right) \wedge \cdots \wedge e_{\alpha_{m}}
$$

where $\left\{e_{\alpha_{1}} \wedge \cdots \wedge e_{\alpha_{m}} \mid 1 \leq \alpha_{1}<\cdots<\alpha_{m} \leq n\right\}$ is the standard basis for $\wedge^{m} \mathbb{R}^{n}$. Then $W$ is the matrix of $U^{[m]}$ under this standard basis. It is convenient to denote the multi-index by $\bar{\alpha}=\left(\alpha_{1} \cdots \alpha_{m}\right)$. We only consider the increasing multi-index, that is, $1 \leq \alpha_{1}<\cdots<\alpha_{m} \leq n$. By the dictionary arrangement, we can arrange all increasing multi-indexes from 1 to $C_{n}^{m}$, and use $N_{\bar{\alpha}}$ denote the order number of the multi-index $\bar{\alpha}=\left(\alpha_{1} \cdots \alpha_{m}\right)$, i.e., $N_{\bar{\alpha}}=1$ for $\bar{\alpha}=(12 \cdots m), \cdots$. We also use $\bar{\alpha}$ denote the index set $\left\{\alpha_{1}, \cdots, \alpha_{m}\right\}$ without confusion. It is not hard to see that

$$
\begin{aligned}
& W_{N_{\bar{\alpha}} N_{\bar{\alpha}}}=w_{\bar{\alpha}, \bar{\alpha}}=\sum_{i=1}^{m} u_{\alpha_{i} \alpha_{i},} \\
& W_{N_{\bar{\alpha}} N_{\bar{\beta}}}=w_{\bar{\alpha} \bar{\beta}}=(-1)^{|i-j|} u_{\alpha_{i} \beta_{j}},
\end{aligned}
$$

if the index set $\left\{\alpha_{1}, \cdots, \alpha_{m}\right\} \backslash\left\{\alpha_{i}\right\}$ equals to the index set $\left\{\beta_{1}, \cdots, \beta_{m}\right\} \backslash\left\{\beta_{j}\right\}$ but $\alpha_{i} \neq \beta_{j}$; and also

$$
W_{N_{\bar{\alpha}} N_{\bar{\beta}}}=w_{\bar{\alpha} \bar{\beta}}=0,
$$

if the index sets $\left\{\alpha_{1}, \cdots, \alpha_{m}\right\}$ and $\left\{\beta_{1}, \cdots, \beta_{m}\right\}$ have more than one different element. Specifically, for $n=3, m=2$, we have

$$
W=\left(\begin{array}{ccc}
u_{11}+u_{22} & u_{23} & -u_{13} \\
u_{32} & u_{11}+u_{33} & u_{12} \\
-u_{31} & u_{21} & u_{22}+u_{33}
\end{array}\right) .
$$

It follows that $W$ is symmetrical and diagonal with $\left(u_{i j}\right)_{n \times n}$ diagonal. The eigenvalues of $W$ are the sums of eigenvalues of $\left(u_{i j}\right)_{n \times n}$. Denoted by $\mu\left(D^{2} u\right)=\left(\mu_{1}, \cdots, \mu_{n}\right)$ the eigenvalues of the Hessian and by $\lambda(W)=\left(\lambda_{1}, \lambda_{2}, \cdots, \lambda_{C_{n}^{m}}\right)$ the eigenvalues of $W$. Generally, for any $k=1,2, \cdots, C_{n}^{m}$, we define the $k^{\text {th }}$ elementary symmetry function by

$$
S_{k}(W)=S_{k}(\lambda(W))=\sum_{1 \leq i_{1}<i_{2}<\cdots<i_{k} \leq C_{n}^{m}} \lambda_{i_{1}} \lambda_{i_{2}} \cdots \lambda_{i_{k}}
$$


We also set $S_{0}=1$. In particular, we have

$$
\operatorname{det}(W)=S_{C_{n}^{m}}(W)=\lambda_{1} \lambda_{2} \cdots \lambda_{C_{n}^{m}}=\prod_{1 \leq i_{1}<i_{2}<\cdots<i_{m} \leq n}\left(\mu_{i_{1}}+\mu_{i_{2}}+\cdots+\mu_{i_{m}}\right) .
$$

If $m=1$, the equation (1.1) is known as Monge-Ampère equation.

Define the Gårding's cone in $\mathbb{R}^{n}$ by

$$
\Gamma_{k, n}=\left\{\mu \in \mathbb{R}^{n} \mid S_{i}(\mu)>0, \forall 1 \leq i \leq k\right\} .
$$

Then we define the generalized Gårding's cone by, for $1 \leq m \leq n, 1 \leq k \leq C_{n}^{m}$,

$$
\Gamma_{k, n}^{(m)}=\left\{\mu \in \mathbb{R}^{n} \mid\left\{\mu_{i_{1}}+\cdots+\mu_{i_{m}} \mid 1 \leq i_{1}<\cdots<i_{m} \leq n\right\} \in \Gamma_{k, C_{n}^{m}}\right\}
$$

In the absence of ambiguity, we omit the subscript $n$ for simplicity. Obviously, $\Gamma_{k}=\Gamma_{k}^{(1)}$ and $\Gamma_{n} \subset \Gamma_{k}^{(m)} \subset \Gamma_{1}$. Normally, we say a $C^{2}$ function $u$ is convex if any eigenvalue of the Hessian is nonnegative, equivalently $\mu\left(D^{2} u\right) \in \overline{\Gamma_{n}}$. Similarly, we give the following definition of $m$-convexity.

Definition 1.1. We say a $C^{2}$ function $u$ is strictly m-convex if $\mu\left(D^{2} u\right) \in \Gamma_{C_{n}^{m}}^{(m)}$, i.e., the sum of any $m$ eigenvalues of the Hessian is positive. Furthermore, we say $u$ is $m$-convex if $\mu\left(D^{2} u\right) \in \overline{\Gamma_{C_{n}^{m}}^{(m)}}$, i.e., the sum of any m eigenvalues of the Hessian is nonnegative.

In particular, if $\mu\left(D^{2} u\right) \in \Gamma_{n}^{(n-1)}$ for any $x \in \Omega$, then equivalently $\lambda(W) \in \Gamma_{n}$, such that the equation (1.1) is elliptic (see [2] or [18]). In addition, we say $u$ is a strictly $(n-1)$ convex solution if $u$ is a solution of (1.1).

For the Dirichlet problem in $\mathbb{R}^{n}$, many results are known. For example, the Dirichlet problem of Laplace equation is studied in [8], Caffarelli-Nirenberg-Spruck [1], and Ivochkina [16] solved the Dirichlet problem of Monge-Ampère equation, and CaffarelliNirenberg-Spruck [2] solved the Dirichlet problem of general Hessian equations even including the case considered here. For the general Hessian quotient equation, the Dirichlet problem is solved by Trudinger in [31]. Finally, Guan [9] treated the Dirichlet problem for general fully nonlinear elliptic equation on the Riemannian manifolds.

Also, the Neumann or oblique derivative problem of partial differential equations was widely studied. For a priori estimates and the existence theorem of Laplace equation with Neumann boundary condition, we refer to the book [8]. Also, we can see the book written by Lieberman [17] for the Neumann or oblique derivative problem of linear and quasilinear elliptic equations. In 1987, Lions-Trudinger-Urbas solved the Neumann problem of Monge-Ampère equation in the celebrated paper [21]. For the the Neumann problem of $k$-Hessian equations, Trudinger [32] established the existence theorem when the domain is a ball, and he conjectured (in [32], page 305) that one can solve the problem in sufficiently smooth uniformly convex domains. Recently, Ma and Qiu [22] gave a positive answer to this problem and solved the the Neumann problem of $k$-Hessian equations 
in uniformly convex domains. After their work, the research on the Neumann problem of other equatios has made progresses (see, e.g., [3, 4, 23, 33]).

Similarly to $m$-convexity for the Hessian (see Definition 1.1), we can formulate the notion of $m$-convexity for curvature operator and second fundamental forms of hypersurfaces. There are large amount literature in differential geometry on this subject. For example, Sha [25] and $\mathrm{Wu}$ [34] introduced the $m$-convexity of the sectional curvature of Riemannian manifolds and studied the topology for these manifolds. In a series interesting papers, Harvey and Lawson ([11-13]) introduce some generalized convexities on the solutions of the nonlinear elliptic Dirichlet problem, $m$-convexity is a special case. Han$\mathrm{Ma}-\mathrm{Wu}[10]$ obtained an existence theorem of $m$-convex starshaped hypersurface with prescribed mean curvature. More recently, in the complex space $\mathbb{C}^{n}$ case, Tosatti and Weinkove $([29,30])$ solved the Monge-Ampère equation for $(n-1)$-plurisubharmonic functions on a compact Kähler manifold, where the $(n-1)$-plurisubharmonicity means the sum of any $n-1$ eigenvalues of the complex Hessian is nonnegative.

From the above geometry and analysis reasons, it is naturally to study the Neumann problem (1.1). In [6], the author considered the following Neumann problem for general fully nonlinear equations

$$
\begin{cases}S_{k}(W)=f(x), & \text { in } \Omega, \\ \frac{\partial u}{\partial v}=-u+\phi(x), & \text { on } \partial \Omega .\end{cases}
$$

Eq. (1.1) is a special case of (1.6) when $m=n-1, k=n$. Parallel to Definition 1.1, we give

Definition 1.2. We say $u$ is $k$-admissible if $\mu\left(D^{2} u\right) \in \Gamma_{k}^{(m)}$. Particularly, if $k=C_{n}^{m}, u$ is strictly m-convex.

For $k \leq C_{n-1}^{m-1}=\frac{m}{n} C_{n}^{m}$, we obtained an existence theorem of the $k$-admissible solution with less geometric restrictions to the boundary. For $m<\frac{n}{2}$ and $k=C_{n-1}^{m-1}+k_{0} \leq \frac{n-m}{n} C_{n}^{m}$, we got an existence theorem if $\Omega$ is strictly $\left(m, k_{0}\right)$-convex, i.e., $\kappa \in \Gamma_{k_{0}}^{(m)}$, where $\kappa=\left(\kappa_{1}, \cdots, \kappa_{n-1}\right)$ denote the principal curvatures of $\partial \Omega$ with respect to its inner normal $-v$. We didn't prove the existence for strictly $m$-convex solution for the equation (1.6) in [6]. Particularly, for $m=n-1$ (maybe the most interesting case except the case $m=1$ ), we got the existence of the $k$-admissible solution for $k \leq n-1$ only except that of the $(n-1)$-convex solution for $k=n$. In this paper, given a strong geometric restriction to the boundary, we can prove the existence of strictly $(n-1)$-convex solution to the Neumann problem (1.1).

We always denote $\kappa=\left(\kappa_{1}, \cdots, \kappa_{n-1}\right)$ the principal curvature and $H=\sum_{i=1}^{n-1} \kappa_{i}$ the mean curvature of the boundary. We now state the main result of this paper as follows.

Theorem 1.1. Suppose $\Omega \subset \mathbb{R}^{n}(n \geq 3)$ is a bounded strictly convex domain with $C^{4}$ boundary. Denote $\kappa_{\max }(x)\left(\kappa_{\min }(x)\right)$ the maximum (minimum) principal curvature at $x \in \partial \Omega$ such that

$$
\kappa_{\max }-\kappa_{\min }<\frac{H}{2(n-1)(n-2)} .
$$


Let $f \in C^{2}(\Omega)$ be a positive function and $\phi \in C^{3}(\bar{\Omega})$. Then there exists a unique strictly $(n-1)$ convex solution $u \in C^{3, \alpha}(\bar{\Omega})$ of the Neumann problem (1.1).

We may point out that the curvature condition (1.7) is only used to obtain the upper bound for the double normal derivative in Lemma 4.3. When the dimension $n$ is large, it is easy to see that the domain $\Omega$ is almost a ball. As a special case, for $n=3, H=\kappa_{\max }+\kappa_{\min }$, we have

Corollary 1.1. Suppose $\Omega \subset \mathbb{R}^{3}$ is a bounded strictly convex domain with $C^{4}$ boundary. Denote $\kappa_{\max }(x)\left(\kappa_{\min }(x)\right)$ the maximum (minimum) principal curvature at $x \in \partial \Omega$ such that $\kappa_{\max }<$ $\frac{5}{3} \kappa_{\min }$. Let $f \in C^{2}(\Omega)$ be a positive function and $\phi \in C^{3}(\bar{\Omega})$. Then there exists a unique strictly 2-convex solution $u \in C^{3, \alpha}(\bar{\Omega})$ of the Neumann problem (1.1).

The rest of this paper is arranged as follows. In Section 2, we give some basic properties of the elementary symmetric functions and some notations. In Section 3, we establish a priori $C^{0}$ estimates and global gradient estimates. In Section 4 , we show the proof of the global estimates of second order derivatives. Finally, we can prove the existence theorem by the method of continuity in Section 5 .

\section{Preliminary}

In this section, we give some basic properties of elementary symmetric functions and some notations. First, we denote by $S_{k}(\lambda \mid i)$ the symmetric function with $\lambda_{i}=0$ and $S_{k}(\lambda \mid i j)$ the symmetric function with $\lambda_{i}=\lambda_{j}=0$.

Proposition 2.1. Let $\lambda=\left(\lambda_{1}, \cdots, \lambda_{n}\right) \in \mathbb{R}^{n}$ and $k=1, \cdots, n$, then

$$
\begin{aligned}
& S_{k}(\lambda)=S_{k}(\lambda \mid i)+\lambda_{i} S_{k-1}(\lambda \mid i), \quad \forall 1 \leq i \leq n, \\
& \sum_{i=1}^{n} \lambda_{i} S_{k-1}(\lambda \mid i)=k S_{k}(\lambda), \\
& \sum_{i=1}^{n} S_{k}(\lambda \mid i)=(n-k) S_{k}(\lambda) .
\end{aligned}
$$

We also denote by $S_{k}(W \mid i)$ the symmetric function with $W$ deleting the $i$-row and $i$ column and $S_{k}(W \mid i j)$ the symmetric function with $W$ deleting the $i, j$-rows and $i, j$-columns. Then we have the following identities.

Proposition 2.2. Suppose $A=\left(a_{i j}\right)_{n \times n}$ is diagonal and $k$ is a positive integer, then

$$
\frac{\partial S_{k}(A)}{\partial a_{i j}}= \begin{cases}S_{k-1}(A \mid i), & \text { if } i=j, \\ 0, & \text { if } i \neq j .\end{cases}
$$


Furthermore, suppose $W=\left(w_{\bar{\alpha} \bar{\beta}}\right)_{C_{n}^{m} \times C_{n}^{m}}$ defined as in (1.2) is diagonal, then

$$
\frac{\partial S_{k}(W)}{\partial u_{i j}}= \begin{cases}\sum_{i \in \bar{\alpha}} S_{k-1}\left(W \mid N_{\bar{\alpha}}\right), & \text { if } i=j, \\ 0, & \text { if } i \neq j .\end{cases}
$$

Proof. For (2.4), see a proof in [18]. Note that

$$
\frac{\partial S_{k}(W)}{\partial u_{i j}}=\sum_{\bar{\alpha}, \bar{\beta}} \frac{\partial S_{k}(W)}{\partial w_{\bar{\alpha} \bar{\beta}}} \frac{\partial w_{\bar{\alpha} \bar{\beta}}}{\partial u_{i j}}
$$

Using (1.3) and (1.4), then (1.5) and (2.5) are immediate consequences of (2.4).

Recall that the Gårding's cone is defined by

$$
\Gamma_{k}=\left\{\lambda \in \mathbb{R}^{n} \mid S_{i}(\lambda)>0, \quad \forall 1 \leq i \leq k\right\} .
$$

Proposition 2.3. Let $\lambda \in \Gamma_{k}$ and $k \in\{1, \cdots, n\}$. Suppose that $\lambda_{1} \geq \cdots \geq \lambda_{k} \geq \cdots \geq \lambda_{n}$, then we have

$$
\begin{aligned}
& S_{k-1}(\lambda \mid n) \geq \cdots \geq S_{k-1}(\lambda \mid k) \geq \cdots \geq S_{k-1}(\lambda \mid 1)>0, \\
& \lambda_{1} S_{k-1}(\lambda \mid 1) \geq \frac{k}{n} S_{k}(\lambda), \\
& S_{k}^{\frac{1}{k}}(\lambda) \text { is concave in } \Gamma_{k} .
\end{aligned}
$$

Proof. All the properties are well-known. For example, see [18] or [15] for a proof of (2.7), [5] or [14] for (2.8) and [2] for (2.9).

The Newton-Maclaurin inequality is as follows:

Proposition 2.4. For $\lambda \in \Gamma_{k}$ and $k>l>0$, we have

$$
\left(\frac{S_{k}(\lambda)}{C_{n}^{k}}\right)^{\frac{1}{k}} \leq\left(\frac{S_{l}(\lambda)}{C_{n}^{l}}\right)^{\frac{1}{T}}
$$

where $C_{n}^{k}=\frac{n !}{k !(n-k) !}$. Furthermore we have

$$
\sum_{i=1}^{n} \frac{\partial S_{k}^{\frac{1}{k}}}{\partial \lambda_{i}} \geq\left[C_{n}^{k}\right]^{\frac{1}{k}}
$$

Proof. See [28] for a proof of (2.10). For (2.11), we use (2.10) and Proposition 2.1 to get

$$
\sum_{i=1}^{n} \frac{\partial S_{k}^{\frac{1}{k}}(\lambda)}{\partial \lambda_{i}}=\frac{1}{k} S_{k}^{\frac{1}{k}-1} \sum_{i=1}^{n} S_{k-1}(\lambda \mid i)=\frac{n-k+1}{k} S_{k}^{\frac{1}{k}-1} S_{k-1}(\lambda) \geq\left[C_{n}^{k}\right]^{\frac{1}{k}}
$$

This completes the proof of the proposition. 
We define

$$
d(x)=\operatorname{dist}(x, \partial \Omega), \quad \Omega_{\mu}=\{x \in \Omega \mid d(x)<\mu\} .
$$

It is well known that there exists a small positive universal constant $\mu_{0}$ such that $d(x) \in$ $C^{4}\left(\Omega_{\mu}\right), \forall 0<\mu \leq \mu_{0}$, provided $\partial \Omega \in C^{4}$. As in Simon-Spruck [27] or Lieberman [17] (p. 331), we can extend $v$ by $v=-D d$ in $\Omega_{\mu}$ and note that $v$ is a $C^{3}\left(\overline{\Omega_{\mu}}\right)$ vector field. As mentioned in the book [17], we also have the following formulas

$$
\begin{array}{ll}
|D v|+\left|D^{2} v\right| \leq C(n, \Omega), & \text { in } \Omega_{\mu}, \\
\sum_{i=1}^{n} v^{i} D_{j} v^{i}=\sum_{i=1}^{n} v^{i} D_{i} v^{j}=\sum_{i=1}^{n} d_{i} d_{i j}=0, & \text { in } \Omega_{\mu}, \\
|v|=|D d|=1, & \text { in } \Omega_{\mu} .
\end{array}
$$

\section{The zero-order and first-order estimates}

As proved in [6], we have the following theorem.

Theorem 3.1. Let $\Omega \subset \mathbb{R}^{n}(n \geq 3)$ be a bounded domain with $C^{3}$ boundary, and $f \in C^{1}(\Omega)$ be a positive function and $\phi \in C^{3}(\bar{\Omega})$. Suppose that $u \in C^{2}(\bar{\Omega}) \cap C^{3}(\Omega)$ is a k-admissible solution of the Neumann problem (1.6). Then there exists a constant $C_{1}$ depending only on $k, m, n,|f|_{C^{1}}$, $|\phi|_{C^{3}}$ and $\Omega$, such that

$$
\sup _{\bar{\Omega}}(|u|+|D u|) \leq C_{1}
$$

Proof. See Theorem 3.1 in [6] for the zero-order estimate. See Theorem 4.2 and Theorem 4.4 in [6] for the first-order estimate. The proof of the gradient estimates could also be found in [7].

\section{Global second order derivatives estimates}

Generally, the double normal estimates are the most important and hardest parts for the Neumann problem. As in [21] and [22], we construct sub and super barrier functions to give lower and upper bounds for $u_{v v}$ on the boundary. Then we give the global second order estimates.

In this section, we establish the following global second order estimate.

Theorem 4.1. Suppose $\Omega \subset \mathbb{R}^{n}(n \geq 3)$ is a bounded strictly convex domain with $C^{4}$ boundary. Denote $\kappa_{\max }(x)\left(\kappa_{\min }(x)\right)$ the maximum (minimum) principal curvature at $x \in \partial \Omega$ such that

$$
\kappa_{\max }-\kappa_{\min }<\frac{\gamma H}{2(n-1)(n-2)} \quad \forall \gamma \in\left[\frac{1}{2}, 1\right) .
$$


Let $f(x, z) \in C^{2}(\Omega \times \mathbb{R})$ be a positive function and $\phi(x, z) \in C^{3}(\bar{\Omega} \times \mathbb{R})$ be decreasing with respect to $z$. If $u \in C^{3, \alpha}(\bar{\Omega})$ is a strictly $(n-1)$-convex solution of the Neumann problem

$$
\begin{cases}\operatorname{det}(W)=f(x, u), & \text { in } \Omega, \\ \frac{\partial u}{\partial v}=\phi(x, u), & \text { on } \partial \Omega .\end{cases}
$$

Then we have

$$
\sup _{\bar{\Omega}}\left|D^{2} u\right| \leq C,
$$

where $C$ depends only on $n, \gamma,|u|_{C^{1}(\bar{\Omega})},|f|_{C^{2}\left(\bar{\Omega} \times\left[-M_{0}, M_{0}\right]\right)}, \min f,|\phi|_{C^{3}\left(\bar{\Omega} \times\left[-M_{0}, M_{0}\right]\right)}$ and $\Omega$, with $M_{0}=\sup _{\Omega}|u|$.

Throughout the rest of this paper, we always admit the Einstein's summation convention. All repeated indices come from 1 to $\mathrm{n}$. We will always denote $F\left(D^{2} u\right)=\operatorname{det}(W)$ and

$$
F^{i j}=\frac{\partial F\left(D^{2} u\right)}{\partial u_{i j}}=\frac{\partial \operatorname{det}(W)}{\partial w_{\bar{\alpha} \bar{\beta}}} \frac{\partial w_{\bar{\alpha} \bar{\beta}}}{\partial u_{i j}} .
$$

From (1.3) and (2.5) in Proposition 2.2 we have, for any $1 \leq i \leq n$,

$$
F^{i i}=\sum_{i \in \bar{\alpha}} \frac{\partial \operatorname{det}(W)}{\partial w_{\overline{\alpha \alpha}}}=\sum_{i \in \bar{\alpha}} S_{n-1}\left(W \mid N_{\bar{\alpha}}\right) .
$$

Throughout the rest of the paper, we will also denote

$$
\mathcal{F}=\sum_{i=1}^{n} F^{i i}=(n-1) \sum_{N_{\bar{\alpha}}=1}^{n} S_{n-1}\left(W \mid N_{\bar{\alpha}}\right)
$$

for simplicity.

\subsection{Reduce the global second derivative estimates into double normal deriva- tives estimates on boundary}

Using the method of Lions-Trudinger-Urbas [21], we can reduce the second derivative estimates of the solution into the boundary double normal estimates.

Lemma 4.1. Let $\Omega \subset \mathbb{R}^{n}$ be a bounded strictly convex domain with $C^{4}$ boundary. Assume $f(x, z) \in C^{2}(\bar{\Omega} \times \mathbb{R})$ is positive and $\phi(x, z) \in C^{3}(\bar{\Omega} \times \mathbb{R})$ is decreasing with respect to $z$. If $u$ is a strictly $(n-1)$-convex solution of the Neumann problem (4.1), denote $N=\sup _{\partial \Omega}\left|u_{v v}\right|$, then we have

$$
\sup _{\bar{\Omega}}\left|D^{2} u\right| \leq C_{0}(1+N)
$$


where $C_{0}$ depends only on $n,|u|_{C^{1}(\bar{\Omega})},|f|_{C^{2}\left(\bar{\Omega} \times\left[-M_{0}, M_{0}\right]\right)}, \min f,|\phi|_{C^{3}\left(\bar{\Omega} \times\left[-M_{0}, M_{0}\right]\right)}$ and $\Omega$, with $M_{0}=\sup _{\Omega}|u|$.

Proof. Write Eq. (4.1) in the form of

$$
\begin{cases}\operatorname{det}(W)^{\frac{1}{n}}=\tilde{f}(x, u), & \text { in } \Omega, \\ \frac{\partial u}{\partial v}=\phi(x, u), & \text { on } \partial \Omega,\end{cases}
$$

where $\widetilde{f}=f^{\frac{1}{n}}$. Since $\lambda(W) \in \Gamma_{n} \subset \Gamma_{2}$ in $\mathbb{R}^{n}$, we have

$$
\sum_{i \neq j}\left|u_{i j}\right| \leq c(n) S_{1}(W)=c(n) S_{1}\left(D^{2} u\right),
$$

where $c(n)$ is a universal number independent of $u$. It is sufficiently to prove (4.5) for any direction $\xi \in \mathbb{S}^{n-1}$, that is

$$
u_{\xi \xi} \leq C_{0}(1+N) .
$$

We consider the following auxiliary function in $\Omega \times \mathrm{S}^{n-1}$,

$$
v(x, \xi)=u_{\xi \xi}-v^{\prime}(x, \xi)+K_{1}|x|^{2}+K_{2}|D u|^{2},
$$

where

$$
v^{\prime}(x, \xi)=a^{l} u_{l}+b=2(\xi \cdot v) \xi^{\prime} \cdot\left(D_{x} \phi+\phi_{z} D u-u_{l} D v^{l}\right),
$$

with $\xi^{\prime}=\xi-(\xi \cdot v) v$ and

$$
a^{l}=2(\xi \cdot v)\left(\xi^{\prime l} \phi_{z}-\xi^{\prime i} D_{i} v^{l}\right), \quad b=2(\xi \cdot v) \xi^{\prime l} \phi_{x_{l}} .
$$

$K_{1}, K_{2}$ are positive constants to be determined. By direct computations, we have

$$
\begin{gathered}
v_{i}=u_{\xi \xi i}-D_{i} a^{l} u_{l}-a^{l} u_{l i}-D_{i} b+2 K_{1} x_{i}+2 K_{2} u_{l} u_{l i}, \\
v_{i j}=u_{\xi \xi i j}-D_{i j} a^{l} u_{l}-D_{i} a^{l} u_{l j}-D_{j} a^{l} u_{l i}-a^{l} u_{l i j}-D_{i j} b \\
+2 K_{1} \delta_{i j}+2 K_{2} u_{l i} u_{l j}+2 K_{2} u_{l} u_{l i j} .
\end{gathered}
$$

Denote $\widetilde{F}\left(D^{2} u\right)=\operatorname{det}(W)^{\frac{1}{n}}$, and

$$
\widetilde{F}^{i j}=\frac{\partial \widetilde{F}}{\partial u_{i j}}=\frac{1}{n} \operatorname{det}(W)^{\frac{1-n}{n}} \frac{\partial \operatorname{det}(W)}{\partial w_{\bar{\alpha} \bar{\beta}}} \frac{\partial w_{\bar{\alpha} \bar{\beta}}}{\partial u_{i j}},
$$

and

$$
\widetilde{F}^{p q, r s}=\frac{\partial^{2} \widetilde{F}}{\partial u_{p q} \partial u_{r s}}=\frac{1}{n} \operatorname{det}(W)^{\frac{1-n}{n}} \frac{\partial^{2} \operatorname{det}(W)}{\partial w_{\bar{\alpha} \bar{\beta}} \partial w_{\bar{\eta} \bar{\zeta}}} \frac{\partial w_{\bar{\alpha} \bar{\beta}}}{\partial u_{p q}} \frac{\partial w_{\bar{\eta} \bar{\xi}}}{\partial u_{r s}}
$$


since $w_{\bar{\alpha} \bar{\beta}}$ is a linear combination of $u_{i j}, 1 \leq i, j \leq n$. Differentiating Eq. (4.6) twice, we have

$$
\begin{aligned}
& \widetilde{F}^{i j} u_{i j l}=D_{l} \widetilde{f}, \\
& \widetilde{F}^{p q, r s} u_{p q \xi} u_{r s \xi}+\widetilde{F}^{i j} u_{i j \xi \xi}=D_{\xi \xi} \widetilde{f} .
\end{aligned}
$$

By the concavity of $\operatorname{det}(W)^{\frac{1}{n}}$ operator with respect to $W$, we have

$$
D_{\xi \xi} \widetilde{f}=\widetilde{F}^{p q, r s} u_{p q \xi} u_{r s \xi}+\widetilde{F}^{i j} u_{i j \xi \xi} \leq \widetilde{F}^{i j} u_{i j \xi \xi} .
$$

Now we contract (4.11) with $\widetilde{F}^{i j}$ to get, using (4.14)-(4.16),

$$
\begin{gathered}
\widetilde{F}^{i j} v_{i j}=\widetilde{F}^{i j} u_{i j \xi \xi}-\widetilde{F}^{i j} D_{i j} a^{l} u_{l}-2 \widetilde{F}^{i j} D_{i} a^{l} u_{l j}-\widetilde{F}^{i j} u_{i j l} a^{l} \\
\quad-\widetilde{F}^{i j} D_{i j} b+2 K_{1} \widetilde{\mathcal{F}}+2 K_{2} \widetilde{F}^{i j} u_{i l} u_{j l}+2 K_{2} \widetilde{F}^{i j} u_{i j l} u_{l} \\
\geq D_{\xi \xi} \widetilde{f}-\widetilde{F}^{i j} D_{i j} a^{l} u_{l}-2 \widetilde{F}^{i j} D_{i} a^{l} u_{i j}-a^{l} D_{l} \widetilde{f}-\widetilde{F}^{i j} D_{i j} b \\
+2 K_{1} \widetilde{\mathcal{F}}+2 K_{2} \widetilde{F}^{i j} u_{i l} u_{j l}+2 K_{2} u_{l} D_{l} \widetilde{f},
\end{gathered}
$$

where $\widetilde{\mathcal{F}}=\sum_{i=1}^{n} \widetilde{F}^{i i}$. Note that

$$
\begin{aligned}
& D_{\xi \xi} \widetilde{f}=\widetilde{f}_{\xi \xi}+2 \widetilde{f}_{\xi z} u_{\xi}+\widetilde{f}_{z} u_{\xi \xi}, \quad D_{i j} a^{l}=2(\xi \cdot v) \xi^{\prime l} \phi_{z z} u_{i j}+r_{i j}^{l}, \\
& D_{i j} b=2(\xi \cdot v) \xi^{\prime l} \phi_{x_{l} z} u_{i j}+r_{i j},
\end{aligned}
$$

with $\left|r_{i j}^{l}\right|,\left|r_{i j}\right| \leq C\left(|u|_{C^{1}},|\phi|_{C^{3}},|\partial \Omega|_{C^{4}}\right)$. At the maximum point $x_{0} \in \Omega$ of $v$, we can assume $\left(u_{i j}\right)_{n \times n}$ is diagonal. It follows that, by the Cauchy-Schwartz inequality,

$$
\begin{aligned}
\widetilde{F}^{i j} v_{i j} & \geq-C\left(\widetilde{\mathcal{F}}+K_{2}+1\right)-C \widetilde{F}^{i i}\left|u_{i i}\right|+\widetilde{f}_{z} u_{\xi \xi}+2 K_{1} \widetilde{\mathcal{F}}+2 K_{2} \widetilde{F}^{i i} u_{i i}^{2} \\
& \geq-C\left(\widetilde{\mathcal{F}}+K_{2}+1\right)+\widetilde{f}_{z} u_{\xi \xi}+2 K_{1} \widetilde{\mathcal{F}}+\left(2 K_{2}-1\right) \widetilde{F}^{i i} u_{i i}^{2},
\end{aligned}
$$

where $C=C\left(|u|_{C^{1}},|\phi|_{C^{3}},|\partial \Omega|_{C^{4},},\left.f\right|_{C^{2}}\right)$.

Assume $u_{11} \geq u_{22} \cdots \geq u_{n n}$, and denote $\lambda_{1} \geq \lambda_{2} \geq \cdots \geq \lambda_{n}$ the eigenvalues of the matrix $\left(w_{\bar{\alpha} \bar{\beta}}\right)_{n \times n}$. It is easy to see

$$
\lambda_{1}=u_{11}+\sum_{i=2}^{n-1} u_{i i} \leq(n-1) u_{11} .
$$

Then we have, by (2.5) in Proposition 2.2 and (2.9) in Proposition 2.3,

$$
\begin{aligned}
\widetilde{F}^{11} u_{11}^{2} & =\sum_{1 \in \bar{\alpha}} \frac{1}{n} \operatorname{det}(W)^{\frac{1-n}{n}} S_{n-1}\left(\lambda \mid N_{\bar{\alpha}}\right) u_{11}^{2} \\
& \geq \frac{1}{(n-1) n} \operatorname{det}(W)^{\frac{1-n}{n}} S_{n-1}(\lambda \mid 1) \lambda_{1} u_{11} \\
& =\frac{1}{(n-1) n} \operatorname{det}(W)^{\frac{1}{n}} u_{11}=\frac{\widetilde{f}}{(n-1) n} u_{11} .
\end{aligned}
$$


We can assume $u_{\xi \xi} \geq 0$, otherwise we have (4.8). Plug (4.19) into (4.18) and use the Cauchy-Schwartz inequality, then

$$
\widetilde{F}^{i i} v_{i i} \geq\left(K_{2}-1\right) \sum_{i=1}^{n} \widetilde{F}^{i i} u_{i i}^{2}+\left(\frac{K_{2} \widetilde{f}}{(n-1) n}+\widetilde{f}_{z}\right) u_{\xi \xi}+\left(2 K_{1}-C\right) \widetilde{\mathcal{F}}-C\left(K_{2}+1\right) .
$$

Choosing $K_{2}=\frac{(n-1) \max \left|f_{z}\right|}{\min f}+1$ and $K_{1}=C\left(K_{2}+2\right)+1$, it follows that

$$
\widetilde{F}^{i i} v_{i i} \geq\left(2 K_{1}-C\right) \widetilde{\mathcal{F}}-C\left(K_{2}+1\right)>0,
$$

since we have $\widetilde{\mathcal{F}} \geq 1$ from (2.11). This implies that $v(x, \xi)$ attains its maximum on the boundary by the maximum principle. Now we assume $\left(x_{0}, \xi_{0}\right) \in \partial \Omega \times \mathbb{S}^{n-1}$ is the maximum point of $v(x, \xi)$ in $\bar{\Omega} \times \mathbb{S}^{n-1}$. Then we consider two cases as follows:

Case1. $\xi_{0}$ is a tangential vector at $x_{0} \in \partial \Omega$.

We directly have $\xi_{0} \cdot v=0, v=-D d, v^{\prime}\left(x_{0}, \xi_{0}\right)=0$, and $u_{\tilde{\xi}_{0}, \xi_{0}}\left(x_{0}\right)>0$. As in [17], we define

$$
c^{i j}=\delta_{i j}-v^{i} v^{j}, \quad \text { in } \Omega_{\mu},
$$

and it is easy to see that $c^{i j} D_{j}$ is a tangential direction on $\partial \Omega$. We compute at $\left(x_{0}, \xi_{0}\right)$. From the boundary condition, we have

$$
u_{l i} v^{l}=\left(c^{i j}+v^{i} v^{j}\right) v^{l} u_{l j}=c^{i j} u_{j} \phi_{z}+c^{i j} \phi_{x_{j}}-c^{i j} u_{l} D_{j} v^{l}+v^{i} v^{j} v^{l} u_{l j} .
$$

It follows that

$$
\begin{aligned}
& u_{l i p} v^{l}=\left[c^{p q}+v^{p} v^{q}\right] u_{l i q} v^{l} \\
= & c^{p q} D_{q}\left(c^{i j} u_{j} \phi_{z}+c^{i j} \phi_{x_{j}}-c^{i j} u_{l} D_{j} v^{l}+v^{i} v^{j} v^{l} u_{l j}\right)-c^{p q} u_{l i} D_{q} v^{l}+v^{p} v^{q} v^{l} u_{l i q},
\end{aligned}
$$

then we obtain

$$
\begin{aligned}
& u_{\tilde{\zeta}_{0} \xi_{0} v}=\sum_{i l p=1}^{n} \xi_{0}^{i} \xi_{0}^{p} u_{l i p} v^{l} \\
= & \sum_{i=1}^{n} \xi_{0}^{i} \xi_{0}^{q}\left(D_{q}\left(c^{i j} u_{j} \phi_{z}+c^{i j} \phi_{x_{j}}-c^{i j} u_{l} D_{j} v^{l}+v^{i} v^{j} v^{l} u_{l j}\right)-u_{l i} D_{q} v^{l}\right) \\
\leq & -2 \xi_{0}^{i} \xi_{0}^{q} u_{l i} D_{q} v^{l}+C\left(1+\left|u_{v v}\right|\right) .
\end{aligned}
$$

We use $\phi_{z} \leq 0$ in the last inequality. We assume $\xi_{0}=e_{1}$, it is easy to get the bound for $u_{1 i}\left(x_{0}\right)$ for $i>1$ from the maximum of $v(x, \xi)$ in the $\xi_{0}$ direction. In fact, we can assume $\xi(t)=\frac{(1, t, 0, \cdots, 0)}{\sqrt{1+t^{2}}}$. Then we have

$$
0=\left.\frac{d v\left(x_{0}, \xi(t)\right)}{d t}\right|_{t=0}=2 u_{12}\left(x_{0}\right)-2 v^{2}\left(\phi_{z} u_{1}-u_{l} D_{l} v^{l}\right),
$$


consequently,

$$
\left|u_{12}\right|\left(x_{0}\right) \leq C+C|D u| .
$$

Similarly, we have for $\forall i>1$,

$$
\left|u_{1 i}\right|\left(x_{0}\right) \leq C+C|D u| .
$$

Thus we have, by $D_{1} v^{1} \geq \kappa_{\min }>0$,

$$
\begin{aligned}
u_{\xi_{0} \xi_{0} v} & \leq-2 D_{1} v^{1} u_{11}+C\left(1+\left|u_{v v}\right|\right) \\
& \leq-2 \kappa_{\min } u_{\xi_{0} \xi_{0}}+C\left(1+\left|u_{v v}\right|\right) .
\end{aligned}
$$

On the other hand, we have from the Hopf lemma, (4.10) and (4.26),

$$
\begin{aligned}
0 & \leq v_{v}\left(x_{0}, \xi_{0}\right) \\
& =u_{\xi_{0} \xi_{0} v}-D_{v} a^{l} u_{l}-a^{l} u_{v v}-D_{v} b+2 K_{1} x_{i} v^{i}+2 K_{2} u_{l} u_{l v} \\
& \leq-2 \kappa_{\min } u_{\xi_{0} \xi_{0}}+C\left(1+\left|u_{v v}\right|\right) .
\end{aligned}
$$

Then we get

$$
u_{\xi_{0} \xi_{0}}\left(x_{0}\right) \leq C\left(1+\left|u_{v v}\right|\right) .
$$

Case2. $\xi_{0}$ is non-tangential.

We can find a tangential vector $\tau$, such that $\xi_{0}=\alpha \tau+\beta v$, with $\alpha^{2}+\beta^{2}=1$. Then we have

$$
\begin{aligned}
u_{\tilde{\zeta}_{0} \xi_{0}}\left(x_{0}\right) & =\alpha^{2} u_{\tau \tau}\left(x_{0}\right)+\beta^{2} u_{v v}\left(x_{0}\right)+2 \alpha \beta u_{\tau v}\left(x_{0}\right) \\
& =\alpha^{2} u_{\tau \tau}\left(x_{0}\right)+\beta^{2} u_{v v}\left(x_{0}\right)+2\left(\xi_{0} \cdot v\right) \xi_{0}^{\prime} \cdot\left(\phi_{z} D u-u_{l} D v^{l}\right) .
\end{aligned}
$$

By the definition of $v\left(x_{0}, \xi_{0}\right)$,

$$
v\left(x_{0}, \xi_{0}\right)=\alpha^{2} v\left(x_{0}, \tau\right)+\beta^{2} v\left(x_{0}, v\right) \leq \alpha^{2} v\left(x_{0}, \xi_{0}\right)+\beta^{2} v\left(x_{0}, v\right)
$$

Thus, $v\left(x_{0}, \xi_{0}\right)=v\left(x_{0}, v\right)$ and

$$
u_{\xi_{0} \xi_{0}}\left(x_{0}\right) \leq\left|u_{v v}\right|+C
$$

In conclusion, we have (4.8) in both cases.

First, we denote $d(x)=\operatorname{dist}(x, \partial \Omega)$, and define

$$
h(x)=-d(x)+K_{3} d^{2}(x) .
$$

The constant $K_{3}$ will be determined later. Then we give the following key lemma. 
Lemma 4.2. Suppose $\Omega \subset \mathbb{R}^{n}$ is a bounded strictly convex domain with $C^{2}$ boundary. Denote $\kappa_{\max }(x)\left(\kappa_{\min }(x)\right)$ the maximum (minimum) principal curvature at $x \in \partial \Omega$. Let $u \in C^{2}(\bar{\Omega})$ is strictly $(n-1)$-convex and $h(x)$ is defined as in (4.29). Then, for any $\gamma \in\left[\frac{1}{2}, 1\right)$, there exists $K_{3}$, a sufficiently large number depending only on $n, \gamma, \min f$ and $\Omega$, such that,

$$
F^{i j} h_{i j} \geq \gamma \kappa_{0}(1+\mathcal{F}), \quad \text { in } \Omega_{\mu}(0<\mu \leq \widetilde{\mu}),
$$

where

$$
\kappa_{0}=\frac{H}{n-1} \geq \kappa_{\min } \text { and } \widetilde{\mu}=\min \left\{\frac{1}{4 K_{3}}, \frac{2-\gamma}{2 K_{3}}, \frac{1}{2 \kappa_{\min }}, \mu_{0}\right\} .
$$

$F^{i j}$ is defined by (4.3), and $\mu_{0}$ is mentioned in (2.13). As $\gamma$ tends to $1, K_{3}$ tends to infinity.

Proof. For $x_{0} \in \Omega_{\mu}$, there exists $y_{0} \in \partial \Omega$ such that $\left|x_{0}-y_{0}\right|=d\left(x_{0}\right)$. Then, in terms of the principal coordinate system at $y_{0}$, we have (see [8], Lemma 14.17)

$$
\begin{aligned}
& {\left[D^{2} d\left(x_{0}\right)\right]=-\operatorname{diag}\left[\frac{\kappa_{1}}{1-\kappa_{1} d}, \cdots, \frac{\kappa_{n-1}}{1-\kappa_{n-1} d}, 0\right],} \\
& \operatorname{Dd}\left(x_{0}\right)=-v\left(x_{0}\right)=(0, \cdots, 0,-1) .
\end{aligned}
$$

Observe that

$$
\left[D^{2} h\left(x_{0}\right)\right]=\operatorname{diag}\left[\frac{\left(\left(1-2 K_{3} d\right) \kappa_{1}\right.}{1-\kappa_{1} d}, \cdots, \frac{\left(1-2 K_{3} d\right) \kappa_{n-1}}{1-\kappa_{n-1} d}, 2 K_{3}\right]
$$

Denote $\mu_{i}=\frac{\left(1-K_{3} d\right) \kappa_{i}}{1-\kappa_{i} d}>0, \forall 1 \leq i \leq n-1$, and $\mu_{n}=2 K_{3}$ for simplicity. Then we define

$$
\lambda\left(D^{2} h\right)=\left\{\mu_{i_{1}}+\cdots+\mu_{i_{n-1}} \mid 1 \leq i_{1}<\cdots<i_{n-1} \leq n\right\}
$$

and assume $\lambda_{1} \geq \cdots \geq \lambda_{n-1} \geq \lambda_{n}$, it is easy to see that

$$
\lambda_{n-1} \geq 2 K_{3}+\sum_{l=1}^{m-1} \mu_{i_{l}} \geq K_{3}
$$

if we choose $K_{3}$ sufficiently large and $\mu \leq \frac{1}{4 K_{3}}$. It is also easy to see that $h$ is strictly convex.

We now consider the function $w=h-\frac{1}{2} \gamma \kappa_{0}|x|^{2}$. As above, we define $\widetilde{\mu}\left(D^{2} w\right)=\left(\widetilde{\mu}_{1}, \cdots, \widetilde{\mu}_{n}\right)$ the eigenvalues of the Hessian $D^{2} w$, and

$$
\widetilde{\lambda}=\left\{\widetilde{\mu}_{i_{1}}+\cdots+\widetilde{\mu}_{i_{n-1}} \mid 1 \leq i_{1}<\cdots<i_{n-1} \leq n\right\}
$$

with $\widetilde{\lambda}_{1} \geq \cdots \geq \widetilde{\lambda}_{n}$. For any $\gamma \in\left[\frac{1}{2}, 1\right)$, assume $\mu \leq \min \left\{\frac{1}{4 K_{3}}, \frac{2-\gamma}{2 K_{3}}, \frac{1}{2 \kappa_{\min }}\right\}$, we have

$$
\frac{1-K_{3} d}{1-\kappa_{i} d}>\gamma, \quad \forall i=1, \cdots, n-1 .
$$


Set $\delta=\frac{1}{2}\left(\frac{1-K_{3} d}{1-\kappa_{\min } d}-\gamma\right)$ independent of $K_{3}$, recalling $H=\sum_{i=1}^{n-1} \kappa_{i}$, it follows that

$$
\widetilde{\lambda}_{n}=\sum_{i=1}^{n-1} \mu_{i}-(n-1) \gamma \kappa_{0} \geq(n-1) \delta \kappa_{0}
$$

By the concavity of $\widetilde{F}\left(D^{2} u\right)=\operatorname{det}(W)^{\frac{1}{n}}$, denote $\widetilde{F}^{i j}=\frac{\partial \widetilde{F}}{\partial u_{i j}}$, we have

$$
\begin{aligned}
\widetilde{F}^{i j} w_{i j} & \geq \widetilde{F}\left[D^{2} u+D^{2} w\right]-\widetilde{F}\left[D^{2} u\right] \geq \widetilde{F}\left[D^{2} w\right] \\
& \geq K_{3}^{n-1}\left((n-1) \delta \kappa_{0}\right) \geq K_{3}
\end{aligned}
$$

for a large enough $K_{3} \geq \frac{1}{(n-1) \delta \kappa_{0}}$. Then we get

$$
\widetilde{F}^{i j} h_{i j}=\widetilde{F}^{i j}\left(h-\frac{1}{2} \gamma \kappa_{0}|x|^{2}+\frac{1}{2} \gamma \kappa_{0}|x|^{2}\right)_{i j} \geq K_{3}+\gamma \kappa_{0} \widetilde{\mathcal{F}} .
$$

If we choose $K_{3} \geq \frac{\gamma \kappa_{0} \max f^{\frac{1}{n}}}{n \min f}$, then we have

$$
F^{i j} h_{i j} \geq \gamma \kappa_{0}(1+\mathcal{F})
$$

This completes the proof of the lemma.

Following the line of Qiu-Ma [22] and Chen-Ma-Zhang [4], we construct the sub barrier function as

$$
P(x)=g(x)(D u \cdot v-\phi(x, u))-G(x),
$$

with

$$
\begin{aligned}
& v(x)=-D d(x), \quad g(x)=1-\beta h(x), \\
& G(x)=(A+\sigma N) h(x),
\end{aligned}
$$

where $A, \sigma$, and $\beta$ are positive constants to be determined. We have the following lemma. The curvature condition in Theorem 4.1 is only used here.

Lemma 4.3. Fix $\sigma$, if we select $\beta$ large, $\mu$ small, A large, and assume $N$ large, then

$$
P \geq 0, \quad \text { in } \Omega_{\mu} \text {. }
$$

Furthermore, we have

$$
\sup _{\partial \Omega} u_{v v} \leq C+\sigma N
$$

where $C$ depends only on $n,|u|_{C^{1}},|\partial \Omega|_{C^{2}}|f|_{C^{2}}$ and $|\phi|_{C^{2}}$. 
Proof. We assume $P(x)$ attains its minimum point $x_{0}$ in the interior of $\Omega_{\mu}$. Differentiate $P$ twice to obtain

$$
\begin{aligned}
P_{i}=g_{i} & \left(u_{l} v^{l}-\phi\right)+g\left(u_{l i} v^{l}+u_{l} D_{i} v^{l}-D_{i} \phi\right)-G_{i}, \\
P_{i j}=g_{i j} & \left(u_{l} v^{l}-\phi\right)+g_{i}\left(u_{l j} v_{l}+u_{l} D_{j} v^{l}-D_{j} \phi\right) \\
& +g_{j}\left(u_{l i} v^{l}+u_{l} D_{i} v^{l}-D_{i} \phi\right)+g\left(u_{l i j} v^{l}+u_{l i} D_{j} v^{l}\right. \\
& \left.+u_{l j} D_{i} v^{l}+u_{l} D_{i j} v^{l}-D_{i j} \phi\right)-G_{i j} .
\end{aligned}
$$

By a rotation of coordinates, we may assume that $\left(u_{i j}\right)_{n \times n}$ is diagonal at $x_{0}$, so are $W$ and $\left(F^{i j}\right)_{n \times n}$, with $F^{i j}$ defined by (4.3).

We choose $\mu<\min \left\{\tilde{\mu}, \frac{2 \epsilon}{\beta}, \frac{\epsilon}{2 K_{3}}\right\}$, where $\tilde{\mu}$ is defined in Lemma 4.2 and $\epsilon \in\left(0, \frac{1}{2}\right)$ is a small positive number to be determined, such that $|\beta h| \leq \beta \frac{\mu}{2} \leq \epsilon$. It follows that

$$
1 \leq g \leq 1+\epsilon
$$

Since $h_{i}=-\left(1-2 K_{3} d\right) d_{i}$, we also have

$$
(1-\epsilon)\left|d_{i}\right| \leq\left|h_{i}\right| \leq\left|d_{i}\right|
$$

By a straight computation, using Lemma 4.2, we obtain

$$
\begin{aligned}
F^{i j} P_{i j}= & F^{i i} g_{i i}\left(u_{l} v^{l}-\phi\right)+2 F^{i i} g_{i}\left(u_{i i} v^{i}+u_{l} D_{i} v^{l}-D_{i} \phi\right) \\
& \quad+g F^{i i}\left(u_{l i i} v^{l}+2 u_{i i} D_{i} v^{i}+u_{l} D_{i i} v^{l}-D_{i i} \phi\right)-(A+\sigma N) F^{i i} h_{i i} \\
\leq & \left(\beta C_{1}-(A+\sigma N) \gamma \kappa_{0}\right)(\mathcal{F}+1)-2 \beta F^{i i} u_{i i} h_{i} v^{i}+2 g F^{i i} u_{i i} D_{i} v^{i},
\end{aligned}
$$

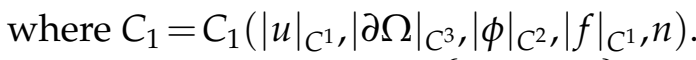

We divide indexes $I=\{1,2, \cdots, n\}$ into two sets in the following way,

$$
B=\left\{i \in I|| \beta d_{i}^{2} \mid<\epsilon \kappa_{\min }\right\}, \quad G=I \backslash B=\left\{i \in I|| \beta d_{i}^{2} \mid \geq \epsilon \kappa_{\min }\right\},
$$

where $\kappa_{\min }\left(\kappa_{\max }\right)$ is the minimum (maximum) principal curvature of the boundary. For $i \in G$, by $P_{i}\left(x_{0}\right)=0$, we get

$$
u_{i i}=\left(1-2 K_{3} d\right)\left(\frac{(A+\sigma N)}{g}+\frac{\beta\left(u_{l} v^{l}-\phi\right)}{g}\right)+\frac{u_{l} D_{i} v^{l}-D_{i} \phi}{d_{i}} .
$$

Because $\left|d_{i}^{2}\right| \geq \frac{\epsilon \kappa_{\min }}{\beta},(4.44)$ and (4.45), we have

$$
\left|\frac{\left(1-2 K_{3} d\right) \beta\left(u_{l} v^{l}-\phi\right)}{g}+\frac{u_{l} D_{i} v^{l}-D_{i} \phi}{d_{i}}\right| \leq \beta C_{2}\left(\epsilon^{-1},|u|_{C^{1},},|\partial \Omega|_{C^{2},|\psi|_{C^{1}}} .\right.
$$

Then let $A \geq 3 \beta C_{2}$, we have

$$
\frac{A}{3}+\frac{1-\epsilon}{1+\epsilon} \sigma N \leq u_{i i} \leq \frac{4 A}{3}+\sigma N,
$$


for $\forall i \in G$. We choose $\beta \geq 2 n \epsilon \mathcal{K}_{\min }+1$ to let $\left|d_{i}^{2}\right| \leq \frac{1}{2 n}$ for $i \in B$. Because $|D d|=1$, there is a $i_{0} \in G$, say $i_{0}=1$, such that

$$
d_{1}^{2} \geq \frac{1}{n}
$$

We have

$$
\begin{aligned}
-2 \beta \sum_{i \in I} F^{i i} u_{i i} h_{i} v^{i} & =-2 \beta \sum_{i \in G} F^{i i} u_{i i} h_{i} v^{i}-2 \beta \sum_{i \in B} F^{i i} u_{i i} h_{i} v^{i} \\
& \leq-2(1-\epsilon) \beta F^{11} u_{11} d_{1}^{2}-2 \beta \sum_{i \in B, u_{i i}<0} F^{i i} u_{i i} d_{i}^{2} \\
& \leq-\frac{\beta F^{11} u_{11}}{n}-2 \epsilon \kappa_{\min } \sum_{u_{i i}<0} F^{i i} u_{i i}, \\
2 g \sum_{i \in I} F^{i i} u_{i i} D_{i} v^{i} & =2 g \sum_{u_{i i} \geq 0} F^{i i} u_{i i} D_{i} v^{i}+2 g \sum_{u_{i i}<0} F^{i i} u_{i i} D_{i} v^{i} \\
& \leq 2 \kappa_{\max } \sum_{u_{i i} \geq 0} F^{i i} u_{i i}+2 \kappa_{\min } \sum_{u_{i i}<0} F^{i i} u_{i i} .
\end{aligned}
$$

Plug (4.50) and (4.51) into (4.46) to get

$$
\begin{aligned}
F^{i j} P_{i j} \leq\left(\beta C_{1}-(A+\sigma N) \gamma \kappa_{0}\right)(\mathcal{F}+1)-\frac{\beta}{2 n} F^{11} u_{11} \\
+2(1-\epsilon) \kappa_{\min } \sum_{u_{i i}<0} F^{i i} u_{i i}+2 \kappa_{\max } \sum_{u_{i i} \geq 0} F^{i i} u_{i i}
\end{aligned}
$$

Denote $u_{22} \geq \cdots \geq u_{n n}$, and

$$
\lambda_{1}=\max _{1 \in \bar{\alpha}}\left\{w_{\overline{\alpha \alpha}}\right\}=u_{11}+\sum_{i=2}^{n-1} u_{i i}, \quad \lambda_{m_{1}}=\min _{1 \in \bar{\alpha}}\left\{w_{\overline{\alpha \alpha}}\right\}=u_{11}+\sum_{i=3}^{n} u_{i i},
$$

and $\lambda_{2} \geq \cdots \geq \lambda_{n}>0$ the eigenvalues of the matrix $W$. Assume $N>1$, from (4.5) we see that

$$
u_{i i} \leq 2 C_{0} N, \quad \forall i \in I
$$

Then

$$
\lambda_{i} \leq 2(n-1) C_{0} N, \quad \forall 1 \leq i \leq n .
$$

If $u_{11} \leq u_{22}$, we see that $\lambda_{m_{1}}=\lambda_{n}$. Then

$$
F^{11}>S_{n-1}(\lambda \mid n) \geq \frac{1}{n(n-1)} \mathcal{F},
$$


it follows that

$$
\begin{gathered}
F^{i j} P_{i j} \leq\left(\beta C_{1}-(A+\sigma N) \gamma \kappa_{0}\right)(\mathcal{F}+1)+2 C_{0} \kappa_{\max } N \mathcal{F} \\
-\frac{\beta}{2 n^{2}(n-1)}\left(\frac{A}{3}+\frac{1-\epsilon}{1+\epsilon} \sigma N\right) \mathcal{F}<0 .
\end{gathered}
$$

if we choose $\beta>12 n^{2}(n-1) \kappa_{\max } C_{0} / \sigma$ and $A>\frac{\beta C_{1}}{\gamma \kappa_{0}}$.

In the following cases, we always assume $u_{11}>u_{22}$.

Case1. $u_{n n} \geq 0$. It follows from

$$
n f=\sum_{i=1}^{n} F^{i i} u_{i i}=\sum_{u_{i i} \geq 0} F^{i i} u_{i i}
$$

and (4.80) that

$$
F^{i j} P_{i j} \leq\left(\beta C_{1}-(A+\sigma N) \gamma \kappa_{0}\right)(\mathcal{F}+1)+2 \kappa_{\max } n f<0,
$$

if we choose $A>\beta C_{1}+2 \kappa_{\max } n \max f / \gamma \kappa_{0}$.

Case2. $B=\sum_{u_{i i}<0} u_{i i}>-(n-2) \sigma N-\epsilon N$ and $\lambda_{n} \leq \epsilon N$. It follows from $\lambda_{n}=\sum_{i=2}^{n} u_{i i}$ that

$$
\begin{aligned}
& 2 \kappa_{\max } \sum_{u_{i i} \geq 0} F^{i i} u_{i i}+(2-\epsilon) \kappa_{\min } \sum_{u_{i i}<0} F^{i i} u_{i i} \\
\leq & 2 \kappa_{\max } F^{11} u_{11}+2\left[\kappa_{\max }(\epsilon N-B)+(1-\epsilon) \kappa_{\min } B\right] \mathcal{F} \\
\leq & 2(n-2)\left[\kappa_{\max }-(1-\epsilon) \kappa_{\min }\right] \sigma N \mathcal{F}+4 \epsilon \kappa_{\max } N \mathcal{F}+2 \kappa_{\max } F^{11} u_{11} .
\end{aligned}
$$

Since $\kappa_{\max }-\kappa_{\min }<\frac{\gamma H}{2(n-1)(n-2)}$, we have

$$
(n-1) \gamma \kappa_{0}=\gamma H \kappa_{\min }>2(n-1)(n-2)\left(\kappa_{\max }-\kappa_{\min }\right) .
$$

We can choose a sufficiently small $\epsilon=\epsilon\left(n, \gamma, \kappa_{\max }, \kappa_{\min }\right)$ to get

$$
2 \kappa_{\max } \sum_{u_{i i} \geq 0} F^{i i} u_{i i}+(2-\epsilon) \kappa_{\min } \sum_{u_{i i}<0} F^{i i} u_{i i} \leq \gamma \kappa_{0} \sigma N \mathcal{F}+2 \kappa_{\max } F^{11} u_{11} \text {. }
$$

We now choose $A>\beta C_{1}+1$ and $\beta \geq 4 n \kappa_{\max }$ to get

$$
F^{i j} P_{i j}<0 \text {. }
$$

Case3. $B=\sum_{u_{i i}<0} u_{i i}>-(n-2) \sigma N-\epsilon N$ and $\lambda_{n}>\epsilon N$. It is easy to see, by (4.54), that,

$$
\begin{aligned}
F^{11} & >S_{n-1}(\lambda \mid 1)=\lambda_{2} \cdots \lambda_{n} \\
& \geq \epsilon^{n-1} N^{n-1}=\left(\frac{\epsilon}{2(n-1) C_{0}}\right)^{n-1}\left[2(n-1) C_{0} N\right]^{n-1} \\
& \geq \frac{1}{n}\left(\frac{\epsilon}{2(n-1) C_{0}}\right)^{n-1} S_{n-1}(\lambda) .
\end{aligned}
$$


Similarly, if we choose $\beta>\frac{2^{n+1} 3 n^{2}(n-1)^{n} \kappa_{\max }\left(C_{0}\right)^{n}}{\sigma \epsilon^{n-1}}$ and $A>\frac{\beta C_{1}}{\gamma \kappa_{0}}$, then

$$
F^{i j} P_{i j}<0
$$

Case4. $B=\sum_{u_{i i}<0} u_{i i} \leq-(n-2) \sigma N-\epsilon N$. We have

$$
\lambda_{n}=u_{22}+\sum_{i=3}^{n} u_{i i}>0
$$

It follows that

$$
u_{22} \geq \frac{|B|}{n-2} \geq\left(\sigma+\frac{\epsilon}{n-2}\right) N>u_{11}
$$

if we assume $N>\frac{4(n-2) A}{3 e}$. This contradicts to that $u_{11}>u_{22}$.

In conclusion, we choose a small $\epsilon=\epsilon\left(n, \gamma, \kappa_{\max }, \kappa_{\min }\right)$,

$$
\beta=\max \left\{4 n \kappa_{\max }+1, \frac{2^{n+1} 3 n^{2}(n-1)^{n} \kappa_{\max }\left(C_{0}\right)^{n}}{\sigma \epsilon^{n-1}}\right\}
$$

and $\mu=\min \left\{\widetilde{\mu}, \frac{2 \epsilon}{\beta}, \frac{\epsilon}{2 K_{3}}\right\}$. If $A>\max \left\{3 \beta C_{2}, \frac{\beta C_{1}+2 \kappa_{\max } n \max f}{\gamma \kappa_{0}}\right\}$ and $N>\frac{4(n-2) A}{3 \epsilon}$, we obtain $F^{i i} P_{i j}<0$, which contradicts to that $P$ attains its minimum in the interior of $\Omega_{\mu}$. This implies that $P$ attains its minimum on the boundary $\partial \Omega_{\mu}$.

On $\partial \Omega$, it is easy to see

$$
P=0 \text {. }
$$

On $\partial \Omega_{\mu} \cap \Omega$, we have

$$
P \geq-C_{3}\left(|u|_{C^{1}},|\phi|_{C^{0}}\right)+(A+\sigma N) \frac{\mu}{2} \geq 0,
$$

if we take $A=\max \left\{\frac{2 C_{3}}{\mu}, 3 \beta C_{2}, \frac{\beta C_{1}+2 \kappa_{\max } n \max f}{\gamma \kappa_{0}}\right\}$. Finally the maximum principle tells us that

$$
P \geq 0, \quad \text { in } \Omega_{\mu} \text {. }
$$

Suppose $u_{v v}\left(y_{0}\right)=\sup _{\partial \Omega} u_{v v}>0$, we have

$$
\begin{aligned}
0 & \geq P_{v}\left(y_{0}\right) \\
& \geq\left(u_{v v}+u_{l} D_{i} v^{l} v^{i}-D_{v} \phi\right)-(A+\sigma N) h_{v} \\
& \geq u_{v v}\left(y_{0}\right)-C\left(|u|_{C^{1}},|\partial \Omega|_{C^{2}},|\phi|_{C^{2}}\right)-(A+\sigma N) .
\end{aligned}
$$

Then we get

$$
\sup _{\partial \Omega} u_{v v} \leq C+\sigma N
$$

This completes the proof of the lemma. 
In a similar way, we construct the super barrier function as

$$
\bar{P}(x):=g(x)(D u \cdot v-\phi(x, u))+G(x) .
$$

We also have the following lemma.

Lemma 4.4. Fix $\sigma$, if we select $\beta$ large, $\mu$ small, $A$ large, then

$$
\bar{P} \leq 0, \quad \text { in } \Omega_{\mu} .
$$

Furthermore, we have

$$
\inf _{\partial \Omega} u_{v v} \geq-C-\sigma N
$$

where $C$ depends only on $n,|u|_{C^{1}},|\partial \Omega|_{C^{2}}|f|_{C^{2}}$ and $|\phi|_{C^{2}}$.

Proof. We assume $\bar{P}(x)$ attains its maximum point $x_{0}$ in the interior of $\Omega_{\mu}$. Differentiate $\bar{P}$ twice to obtain

$$
\begin{aligned}
\bar{P}_{i}=g_{i} & \left(u_{l} v^{l}-\phi\right)+g\left(u_{l i} v^{l}+u_{l} D_{i} v^{l}-D_{i} \phi\right)+G_{i}, \\
\bar{P}_{i j}=g_{i j} & \left(u_{l} v^{l}-\phi\right)+g_{i}\left(u_{l j} v_{l}+u_{l} D_{j} v^{l}-D_{j} \phi\right) \\
& +g_{j}\left(u_{l i} v^{l}+u_{l} D_{i} v^{l}-D_{i} \phi\right)+g\left(u_{l i j} v^{l}+u_{l i} D_{j} v^{l}\right. \\
& \left.+u_{l j} D_{i} v^{l}+u_{l} D_{i j} v^{l}-D_{i j} \phi\right)+G_{i j} .
\end{aligned}
$$

As before we assume that $\left(u_{i j}\right)$ is diagonal at $x_{0}$, so are $W$ and $\left(F^{i j}\right)$, with $F^{i j}$ defined by (4.3). We choose $\mu$ small enough such that $|\beta h| \leq \beta \frac{\mu}{2} \leq \frac{1}{2}$. It follows that

$$
1 \leq g \leq \frac{3}{2}
$$

Recall that $h_{i}=-\left(1-2 K_{3} d\right) d_{i}$, we also have

$$
\frac{1}{2}\left|d_{i}\right| \leq\left|h_{i}\right| \leq\left|d_{i}\right|
$$

By a straight computation, using Lemma 4.7, we obtain

$$
\begin{aligned}
F^{i j} \bar{P}_{i j}= & F^{i i} g_{i i}\left(u_{l} v^{l}-\phi\right)+2 F^{i i} g_{i}\left(u_{i i} v^{i}+u_{l} D_{i} v^{l}-D_{i} \phi\right) \\
& \quad+g F^{i i}\left(u_{l i i} v^{l}+2 u_{i i} D_{i} v^{i}+u_{l} D_{i i} v^{l}-D_{i i} \phi\right)+(A+\sigma N) F^{i i} h_{i i} \\
\geq & \left((A+\sigma N) \gamma \kappa_{0}-\beta C_{1}\right)(\mathcal{F}+1)-2 \beta F^{i i} u_{i i} h_{i} v^{i}+2 g F^{i i} u_{i i} D_{i} v^{i}
\end{aligned}
$$

where $C_{1}=C_{1}\left(|u|_{C^{1},},\left.\left.\partial \Omega\right|_{C^{3}, \mid}|\phi|_{C^{2},|f|}\right|_{\left.C^{1}, n\right)}\right.$.

We divide indexes $I=\{1,2, \cdots, n\}$ into two sets in the following way,

$$
B=\left\{i \in I|| \beta d_{i}^{2} \mid<\frac{1}{2} \kappa_{\min }\right\}, \quad G=I \backslash B=\left\{i \in I|| \beta d_{i}^{2} \mid \geq \frac{1}{2} \kappa_{\min }\right\},
$$


where $\kappa_{\min }\left(\kappa_{\max }\right)$ is the minimum (maximum) principal curvature of the boundary. For $i \in G$, by $\bar{P}_{i}\left(x_{0}\right)=0$, we get

$$
u_{i i}=\left(1-2 K_{3} d\right)\left(\frac{-(A+\sigma N)}{g}+\frac{\beta\left(u_{l} v^{l}-\phi\right)}{g}\right)+\frac{u_{l} D_{i} v^{l}-D_{i} \phi}{d_{i}} .
$$

Because $\left|d_{i}^{2}\right| \geq \frac{\kappa_{\min }}{2 \beta}$, by (4.72) and (4.73), we have

$$
\left|\frac{\left(1-2 K_{3} d\right) \beta\left(u_{l} v^{l}-\phi\right)}{g}+\frac{u_{l} D_{i} v^{l}-D_{i} \phi}{d_{i}}\right| \leq \beta C_{2}\left(|u|_{C^{1}},|\partial \Omega|_{C^{2}},|\psi|_{C^{1}}\right) .
$$

Then let $A \geq 3 \beta C_{2}$, we have

$$
-\frac{4 A}{3}-\sigma N \leq u_{i i} \leq-\frac{A}{3}-\frac{1}{3} \sigma N,
$$

for $\forall i \in G$. We choose $\beta \geq n \kappa_{\min }+1$ to let $\left|d_{i}^{2}\right| \leq \frac{1}{2 n}$ for $i \in B$. Because $|D d|=1$, there is a $i_{0} \in G$, say $i_{0}=1$, such that

$$
d_{1}^{2} \geq \frac{1}{n}
$$

We have

$$
\begin{aligned}
-2 \beta \sum_{i \in I} F^{i i} u_{i i} h_{i} v^{i} & =-2 \beta \sum_{i \in G} F^{i i} u_{i i} h_{i} v^{i}-2 \beta \sum_{i \in B} F^{i i} u_{i i} h_{i} v^{i} \\
& \geq-\beta F^{11} u_{11} d_{1}^{2}-2 \beta \sum_{i \in B, u_{i i}>0} F^{i i} u_{i i} d_{i}^{2} \\
& \geq-\frac{\beta F^{11} u_{11}}{n}-\kappa_{\min } \sum_{u_{i i}>0} F^{i i} u_{i i}, \\
2 g \sum_{i \in I} F^{i i} u_{i i} D_{i} v^{i} & =2 g \sum_{u_{i i}>0} F^{i i} u_{i i} D_{i} v^{i}+2 g \sum_{u_{i i} \leq 0} F^{i i} u_{i i} D_{i} v^{i} \\
& \geq 2 \kappa_{\min } \sum_{u_{i i}>0} F^{i i} u_{i i}+2 \kappa_{\max } \sum_{u_{i i} \leq 0} F^{i i} u_{i i} .
\end{aligned}
$$

Plug (4.78) and (4.79) into (4.74) to get

$$
\begin{gathered}
F^{i i} \bar{P}_{i j} \geq\left(\left(A+\sigma N-\beta C_{1}\right) \gamma \kappa_{0}\right)(\mathcal{F}+1)-\frac{\beta}{2 n} F^{11} u_{11} \\
+\kappa_{\min } \sum_{u_{i i}>0} F^{i i} u_{i i}+2 \kappa_{\max } \sum_{u_{i i} \leq 0} F^{i i} u_{i i} .
\end{gathered}
$$

Denote $u_{22} \geq \cdots \geq u_{n n}$, and

$$
\lambda_{m_{1}}=\min _{1 \in \bar{\alpha}}\left\{w_{\overline{\alpha \alpha}}\right\}=u_{11}+\sum_{i=3}^{n} u_{i i}
$$


and $\lambda_{1} \geq \lambda_{2} \geq \cdots \geq \lambda_{n}>0$ the eigenvalues of the matrix $W$. Assume $N>1$, from (4.5) we see that

$$
u_{i i} \leq 2 C_{0} N, \quad \forall i \in I
$$

Then

$$
\lambda_{i} \leq 2(n-1) C_{0} N, \quad \forall 1 \leq i \leq n .
$$

Since $u_{11} \leq u_{22}$, we see that $\lambda_{m_{1}}=\lambda_{n}$. Then

$$
F^{11}>S_{n-1}(\lambda \mid n) \geq \frac{1}{n(n-1)} \mathcal{F}
$$

it follows that

$$
\begin{gathered}
F^{i j} \bar{P}_{i j} \geq\left((A+\sigma N) \gamma \kappa_{0}-\beta C_{1}\right)(\mathcal{F}+1)-2 C_{0} \kappa_{\max } N \mathcal{F} \\
+\frac{\beta}{2 n^{2}(n-1)}\left(\frac{A}{3}+\frac{1}{3} \sigma N\right) \mathcal{F}>0 .
\end{gathered}
$$

if we choose $\beta=12 n^{2}(n-1) \kappa_{\max } C_{0} / \sigma+n \kappa_{\min }+1$ and $A>\frac{\beta C_{1}}{\gamma \kappa_{0}}$. This contradicts to that $\bar{P}$ attains its maximum in the interior of $\Omega_{\mu}$. This contradiction implies that $\bar{P}$ attains its maximum on the boundary $\partial \Omega_{\mu}$.

On $\partial \Omega$, it is easy to see $\bar{P}=0$. On $\partial \Omega_{\mu} \cap \Omega$, we have

$$
\bar{P} \leq C_{3}\left(|u|_{C^{1}},|\phi|_{C^{0}}\right)-(A+\sigma N) \frac{\mu}{2} \leq 0,
$$

if we take $A=\frac{2 C_{3}}{\mu}+\frac{\beta C_{1}}{k_{3}}+1$. Finally the maximum principle tells us that

$$
\bar{P} \leq 0, \quad \text { in } \Omega_{\mu} .
$$

Suppose $u_{v v}\left(y_{0}\right)=\inf _{\partial \Omega} u_{v v}$, we have

$$
\begin{aligned}
0 & \leq P_{v}\left(y_{0}\right) \\
& \leq\left(u_{v v}+u_{l} D_{i} v^{l} v^{i}-D_{v} \phi\right)+(A+\sigma N) h_{v} \\
& \leq u_{v v}\left(y_{0}\right)+C\left(|u|_{C^{1}},|\partial \Omega|_{C^{2}},|\phi|_{C^{2}}\right)+(A+\sigma N) .
\end{aligned}
$$

Then we get

$$
\inf _{\partial \Omega} u_{v v} \geq-C-\sigma N
$$

This completes the proof of the lemma.

Then we prove Theorem 4.1 immediately.

Proof of Theorem 4.1. We choose $\sigma=\frac{1}{2}$ in Lemmas 4.3 and 4.4, then

$$
\sup _{\partial \Omega}\left|u_{v v}\right| \leq C
$$

Combining (4.88) with (4.5) in Lemma 4.1, we obtain (4.2). 


\section{Existence of the Neumann boundary problem}

We use the method of continuity to prove the existence theorem for the Neumann problem (1.1).

Proof of Theorem 1.1. Consider a family of equations with parameter $t$,

$$
\begin{cases}\operatorname{det}(W)=t f+(1-t)(n-1)^{n}, & \text { in } \Omega, \\ u_{v}=-u+t \phi+(1-t)\left(x \cdot v+\frac{1}{2}|x|^{2}\right), & \text { on } \partial \Omega .\end{cases}
$$

From Theorems 3.1 and 4.1, we get a global $C^{2}$ estimate independent of $t$ for Eq. (5.1). It follows that Eq. (5.1) is uniformly elliptic. Due to the concavity of $\operatorname{det}^{\frac{1}{n}}(W)$ with respect to $D^{2} u$ (see [2]), we can get the global Hölder estimates of second derivatives following the discussions in [19], that is, we can get

$$
|u|_{C^{2, \alpha}} \leq C,
$$

where $C$ depends only on $n,|u|_{C^{2}},|f|_{C^{2}, \min } f,|\phi|_{C^{3}}$ and $\Omega$. It is easy to see that $\frac{1}{2}|x|^{2}$ is a strictly $(n-1)$-convex solution to (5.1) for $t=0$. Applying the method of continuity (see [8], Theorem 17.28), the existence of the classical solution holds for $t=1$. By the standard regularity theory of uniformly elliptic partial differential equations, we can obtain the higher regularity. The uniqueness is easy to get from maximum principle.

\section{Acknowledgments}

The author would like to thank Professor Xi-Nan Ma, his supervisor, for his constant encouragement and guidance. The research of the author is partly supported by NSFC No.11721101 and No.11871255.

\section{References}

[1] Caffarelli L, Nirenberg L, Spruck J. Dirichlet problem for nonlinear second order elliptic equations I. Monge-Ampère equations, Comm Pure Appl Math, 1984, 37: 369-402.

[2] Caffarelli L, Nirenberg L, Spruck J. Dirichlet problem for nonlinear second order elliptic equations III, Functions of the eigenvalues of the Hessian. Acta Math, 1985, 155: 261-301.

[3] Chen C, Ma X, Wei W. The Neumann problem of special Lagrangian equations with supercritical phase, J Differ Equ, 2019, 267(9): 5388-5409.

[4] Chen C, Ma X, Zhang D, The Neumann problem of Parabolic Hessian quotient equations, arXiv: 2001.01427.

[5] Chou K, Wang X. A variation theory of the Hessian equation. Comm Pure Appl Math, 2001, 54(9): 1029-1064.

[6] Deng B. The Neumann problem for a class of fully nonlinear elliptic partial differential equations, arXiv:1903.04231. 
[7] Dong W. Interior and boundary gradient estimates for solutions to Hessian equations satisfying Neumann boundary conditions. Nonlinear Anal, 2020, 195: 111733.

[8] Gilbarg D, Trudinger N. Elliptic Partial Differential Equations of Second Order, SpringerVerlag, Berlin-New York, 1977.

[9] Guan B. Second-order estimates and regularity for fully nonlinear elliptic equations on Riemannian manifolds, Duke Math J, 2014, 163(8): 1491-1524.

[10] Han F, Ma X, Wu D. The existence of k-convex hypersurface with prescribed mean curvature. Calc Var Partial Diffe Equ, 2011, 42(1-2): 43-72.

[11] Harvey F, Lawson H B. Geometric plurisubharmonicity and convexity: an introduction. Adv Math, 2012, 230(4-6): 2428-2456.

[12] Harvey F R, Lawson H B. Dirichlet duality and the nonlinear Dirichlet problem on Riemannian manifolds. J Differ Geom, 2011, 88(3): 395-482.

[13] Harvey F R, Lawson H B. p-convexity, p-plurisubharmonicity and the Levi problem. Indiana Univ Math J, 2013, 62(1): 149-169.

[14] Hou Z, Ma X, Wu D. A second order estimate for complex Hessian equations on a compact Kähler manifold. Math Res Lett, 2010, 17(3): 547-561.

[15] Huisken G, Sinestrari C. Convexity estimates for mean curvature flow and singularities of mean convex surfaces. Acta Math, 1999, 183(1): 45-70.

[16] Ivochkina N. Solutions of the Dirichlet problem for certain equations of Monge-Ampère type (in Russian). Mat Sb, 1985, 128: 403-415.

[17] Lieberman G. Oblique Boundary Value Problems for Elliptic Equations, World Scientific, 2013.

[18] Lieberman G. Second Order Parabolic Differential Equations, World Scientific, 1996.

[19] Lieberman G, Trudinger N. Nonlinear oblique boundary value problems for nonlinear elliptic equations. Trans Amer Math Soc, 1986, 295(2): 509-546.

[20] Lin M, Trudinger N. On some inequalities for elementary symmetric functions. Bull Austral Math Soc, 1994, 50: 317-326.

[21] Lions P, Trudinger N, Urbas J. The Neumann problem for equations of Monge-Ampère type. Comm Pure Appl Math, 1986, 39: 539-563.

[22] Ma X, Qiu G. The Neumann problem for Hessian equations. Comm Math Phys, 2019, 366(1): $1-28$.

[23] $\mathrm{Ma} X, \mathrm{Xu}$ J. Gradient estimates of mean curvature equations with Neumann boundary value problems. Adv Math, 2016, 290: 1010-1039.

[24] Qiu G, Xia C. Classical Neumann problems for Hessian equations and Alexandrov-Fenchel's inequalities. Int Math Res Not IMRN, 2019, 20: 6285-6303.

[25] Sha J. p-convex Riemannian manifolds. Invent Math,1986, 83(3): 437-447.

[26] Sheng W, Trudinger N, Wang X. Prescribed Weingarten curvature equations. Recent Development in Geometry and Analysis, ALM, 2012, 23: 359-386.

[27] Simon L, Spruck J. Existence and regularity of a capillary surface with prescribed contact angle. Arch Rational Mech Anal, 1976, 61: 19-34.

[28] Spruck J. Geometric aspects of the theory of fully nonlinear elliptic equations. Clay Math Proc, 2005, 2: 283-309.

[29] Tosatti $V$, Weinkove $B$. The Monge-Ampère equation for $(n-1)$-plurisubharmonic functions on a compact Kähler manifold. J Amer Math Soc, 2017, 30: 311-346.

[30] Tosatti $V$, Weinkove B. Hermitian metrics, $(n-1, n-1)$-forms and Monge-Ampère equations. J Reine Angew Math, 2019, 755: 67-101.

[31] Trudinger N S. On the Dirichlet problem for Hessian equations. Acta Math, 1995, 175: 151- 
164.

[32] Trudinger N S. On degenerate fully nonlinear elliptic equations in balls. Bulletin of the Australian Math Soc, 1987, 35: 299-307.

[33] Wang J. The Neumann problem of special Lagrangian equations with critical phase. Commun Math Stat, to appear.

[34] Wu H. Manifolds of partially positive curvature. Indiana Univ Math J, 1987, 36: 525-548. 